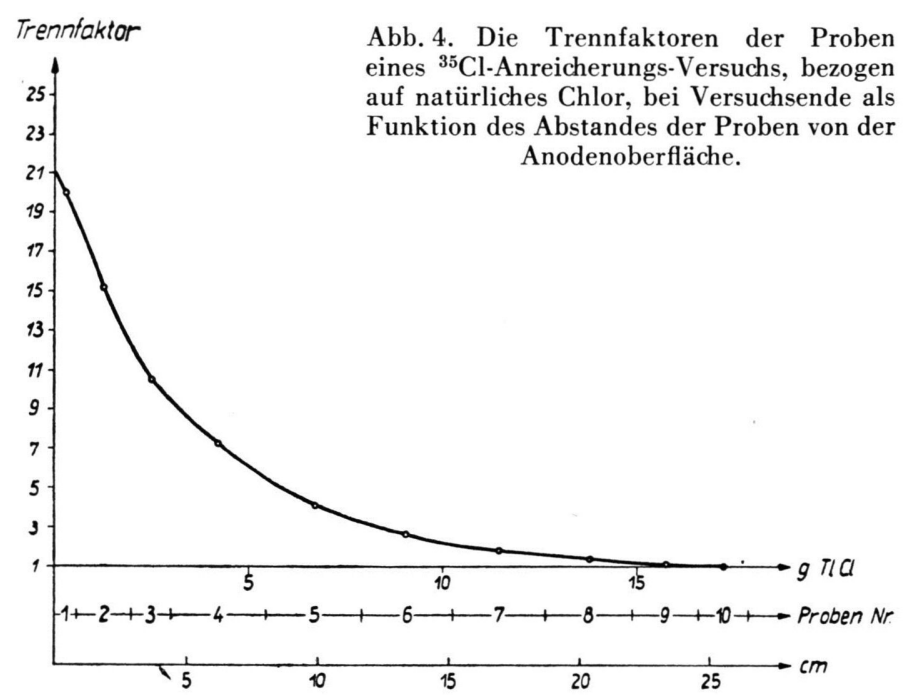

Trennfaktor in Abhängigkeit von der Trennrohrlänge.

Den Herren Glasbläsermeistern P. Kasper und E. Gleichmann sowie Herrn Chemo-Techniker D. Nehring danken wir für ihre unentbehrliche Mitarbeit.

Die Duisburger Kupfer$\mathrm{h} \ddot{\mathrm{u}} \mathrm{t}$ t e, Duisburg, hat uns in dankenswerter Weise die für die Arbeit benötigten Mengen an Thallium-Metall und Thallium(I) chlorid kostenlos zur Verfügung gestellt.

Der Deutschen Forschungsg e meinschaft und dem Bundesministerium für Atomfragen danken wir für die Beihilfen, mit denen sie die Arbeit gefördert haben.

\title{
Massenspektrometrische Untersuchungen von Kohlenwasserstoffen bei hohen Temperaturen
}

\author{
Von H. Ehrhardt und O. Osberghaus \\ Aus dem Physikalischen Institut der Universität Bonn \\ (Z. Naturforschg. 13 a, 16-21 [1958]; eingegangen am 2. November 1957)
}

\begin{abstract}
Es werden die Massenpektren von neun gesättigten Kohlenwasserstoffen mit 5, 6 und 7 C-Atomen im Temperaturgebiet zwischen $100^{\circ} \mathrm{C}$ und $900^{\circ} \mathrm{C}$ untersucht. Zur Messung können die Wände des Ionisierungsraums der Ionenquelle, in dem sich das zu untersuchende Gas bei einem Druck von etwa $10^{-5}$ Torr befindet, bis auf ca. $1000^{\circ} \mathrm{C}$ hochgeheizt werden. Als Wandmaterial diente Gold. Bei einem der Kohlenwasserstoffe (2,2-Dimethylbutan) wurde zusätzlich gemessen, wie sich das Spektrum bei Auskleidung der Wände des Ionisierungsraumes mit einem Crack-Katalysator ändert. Dabei konnte die katalysierte Methan-Bildung als Funktion der Temperatur quantitativ bestimmt werden.
\end{abstract}

Die Massenspektren von Molekülen ändern sich mit der Temperatur ${ }^{1,2,3}$. Diese Temperaturabhängigkeit der Spektren ist eine Folge der mit der Temperatur zunehmenden Besetzung von angeregten Schwingungsniveaus des Moleküls. Bei größeren organischen Molekülen mit tiefliegenden Schwingungstermen können sich die relativen Intensitäten einzelner Massen über einen Temperaturbereich von einigen hundert Grad um Größenordnungen ändern.

1 D. P. Stevenson, J. Chem. Phys. 17, 101 [1949].

2 C. E. Berry, J. Chem. Phys. 17, 1164 [1949].
Die Erscheinung ist aus folgenden Gründen von Interesse:

1. In allen Fällen, in denen ein Massenspektrometer als Analysengerät für die Untersuchung von Reaktionsabläufen bei erhöhter Temperatur benutzt wird, ist die Kenntnis der Spektren als Funktion der Temperatur Voraussetzung für die Analyse. Ein Beispiel hierfür ist die Messung der bei der thermischen Spaltung von Molekülen auftretenden Radi-

3 O. Osberghaus u. R. Taubert, Z. Phys. Chem. 4, 264 [1955]. 
kale $^{3,4,5,6,7,8}$ oder die am Ende dieser Arbeit beschriebene Messung der Methankonzentration bei der Zersetzung eines schweren Kohlenwasserstoffs an der Oberfläche eines Katalysators.

2. Man kann das Massenspektrum als quantitatives Maß für die Anregung von inneren Freiheitsgraden. des Moleküls benutzen, das heißt, seine „Schwingungstemperatur“ messen. Eine Anwendungsmöglichkeit unter vielen ist die Bestimmung des Akkommodationskoeffizienten der Schwingungsenergie beim Wandstoß eines Moleküls.

3. Eine genaue Analyse der Temperaturabhängigkeit des Spektrums kann Anhaltspunkte für den Mechanismus des Molekülzerfalls bei Elektronenstoß geben, wenn zum Beispiel das Auftreten bestimmter Spaltprodukte der Anregung von bestimmten Normalschwingungen im Elektronengrundzustand zugeordnet werden kann.

\section{Versuchsanordnung und Meßverfahren}

Massenspektrometer und Ionenquelle sind in einer früheren Arbeit ${ }^{3}$ beschrieben. Die Wände des etwa $1 \mathrm{~cm}^{3}$ großen Ionisierungsraumes der Quelle (im folgenden als „Ionenkästchen“ bezeichnet), in dem die Ionisierung der Moleküle durch Elektronenbeschuß erfolgt, sind auf etwa $1000^{\circ} \mathrm{C}$ aufheizbar. Das bis auf die Eintrittsöffnung für die Elektronen und den Austrittsspalt der Ionen allseitig geschlossene Ionenkästchen besteht aus Chrom-Nickel, kann aber mit beliebigem Wandmaterial, in diesem Fall mit Gold, ausgekleidet werden. Die Gasmoleküle treten durch ein Zuleitungsrohr in den Ionisierungsraum ein, machen eine Reihe von Wandstößen bei einem Druck von etwa $10^{-5}$ Torr, verlassen schließlich das Ionenkästchen durch eine der Öffnungen und werden bei einem Gasdruck von $10^{-7}$ bis $10^{-6}$ Torr im Spektrometerrohr abgesaugt.

Die mittlere Zahl $\bar{n}$ der Wandstöße im Ionisierungsraum ist annähernd gleich dem Verhältnis von Wandfläche zur Fläche der Austrittsöffnungen und in unserem Fall etwa $60 . \bar{n}$ darf nicht zu klein sein, wenn Temperaturgleichgewicht herrschen soll, weil mit der Möglichkeit gerechnet werden muß, daß die Akkommodationskoeffizienten für die Schwingungsenergie erheblich kleiner als eins sind. Definieren wir den Akkommodationskoeffizienten a durch $\alpha=\left(T-T_{0}\right) /\left(T_{\mathrm{w}}-T_{0}\right)$, mit $T_{0}=$ Schwingungstemperatur der Moleküle vor einem Wandsto $ß, T_{\mathrm{w}}=$ Wandtemperatur und $T$ =Schwingungstemperatur nach

4 G. C. Eltenton, J. Chem. Phys. 15, 455 [1947].

5 A.J.B. Robertson, Proc. Roy. Soc., Lond. A 199, 394 [1949].

6 F.P.Lossing u. A. W. Tickner, J. Chem. Phys. 20,907 [1952], dem Stoß, so gilt unter der Voraussetzung $\alpha \ll 1$ für $T$, die mittlere Schwingungstemperatur der Moleküle im Augenblick der Ionisation durch Elektronenstoß:

$$
\left(T_{\mathrm{w}}-T_{\mathrm{i}}\right) /\left(T_{\mathrm{w}}-T_{0}\right) \approx 1 /\left(1+\bar{n} \alpha_{\mathrm{i}}\right) .
$$

Unter $T_{0}$ ist hier die Temperatur der Moleküle vor Eintritt in das Ionenkästchen, also Raumtemperatur, zu verstehen. Durch den Index i bei $T$ und $\alpha$ soll angedeutet werden, daß es unter Umständen notwendig ist, zwischen den Temperaturen von einzelnen Schwingungsfreiheitsgraden bzw. Symmetriegruppen von Schwingungsfreiheitsgraden zu unterscheiden, weil deren Akkommodationskoeffizienten wahrscheinlich ganz unterschiedlich sein können. Zur vollständigen Einstellung des Temperaturgleichgewichts muß $\bar{n} \alpha_{\mathrm{i}} \gg 1$ sein. Ist die Bedingung $\bar{n} \alpha_{\mathrm{i}} \gg 1$ nicht erfüllt, so kann umgekehrt aus der Änderung des Massenspektrums mit der mittleren Stoßzahl bei konstanter Wandtemperatur (das erfordert eine Änderung der Geometrie des Ionenkästchens, zum Beispiel der Größe der Austrittsöffnungen für die Gasmoleküle) auf die Größe der Akkommodationskoeffizienten geschlossen werden. Über die Werte von $\alpha$ liegen in unserem Temperaturbereich anscheinend keine Messungen vor. Ein Vergleich der mit Ionenkästchen verschiedener mittlerer Stoßzahl gewonnenen Spektren gibt jedoch Anhaltspunkte dafür, daß die $\alpha_{i}$ nicht kleiner als 0,1 sind. Mit einer mittleren Stoßzahl von $\bar{n}=60$ lassen sich aus der oben angegebenen Formel die maximalen Abweichungen zwischen Wandtemperatur und Schwingungstemperatur des Moleküls abschätzen.

Um apparative Einflüsse auf die Temperaturabhängigkeit der Spektren möglichst weitgehend auszuschalten, werden die Ionenströme auf den einzelnen Massen nicht absolut, sondern stets im Verhältnis zum Ionenstrom eines gleichzeitig zugeführten Edelgases gemessen. Unter der Voraussetzung molekularer Strömung im gesamten Strömungssystem des Massenspektrometers verhalten sich die Dichten zweier Gase im Ionisierungsraum für alle Temperaturen wie ihre Dichten im Gasvorratsbehälter. Durch die Relativmessung der Ionenströme werden außerdem geringfügige Änderungen in der Geometrie der Ionenquelle, die infolge der thermischen Ausdehnung der Halterungen des Ionenkästchens nicht ganz zu vermeiden sind, weitgehend

7 P. Le Goff, J. Chim. Phys. 50, 423 [1953].

8 C. A. McDowell, F. P. Lossing, I. H. S. Henderson u. J. B. F ARMer, Canad. J. Chem. 34, 345 [1956]. 
unschädlich gemacht. Abb. 1 gibt einen Eindruck von der erreichbaren Meßgenauigkeit. Die Ionisierungsquerschnitte der Edelgase hängen nicht von der Temperatur ab, die Meßpunkte der Abb. 1 müßten also exakt auf einer Geraden liegen, die parallel zur Abszisse verläuft.

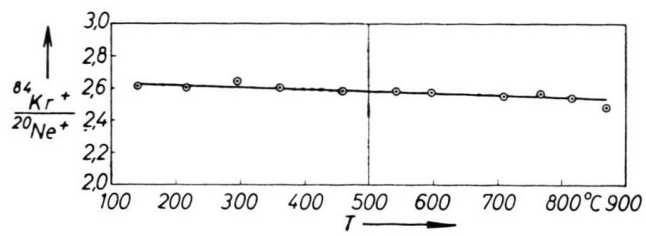

Abb. 1. Verhältnis der Ionenströme von ${ }^{84} \mathrm{Kr}^{+}$und ${ }^{20} \mathrm{Ne}^{+}$als Funktion der Temperatur.

Für die Güte der Messungen sind zwei Bedingungen maßgebend:

1. Die Aufhängung des Ionenkästchens muß an möglichst kurzen Halterungen erfolgen und so stabil sein, daß die Auswanderung des Ionenaustrittsspaltes aus seiner normalen Lage auch bei den höchsten Temperaturen kleiner als $0,1 \mathrm{~mm}$ bleibt.

2. Alle Spalte und Schlitze in den Elektroden des ionenoptischen Systems der Quelle einschließlich des Ioneneintrittsspaltes in das Massenspektrometerrohr müssen so weit sein, daß sie bei geeigneter Wahl der Potentiale nicht von Ionen getroffen werden. Der Querschnitt des Ionenbündels ist dann an jeder Stelle allein durch die Fläche des Ionenaustrittsspaltes im Ionenkästchen bestimmt und die Größe des Ionenstromes im Spektrometerrohr damit unabhängig von geringfügigen Verschiebungen des Kästchens.

Wie stets bei massenspektrometrischen Messungen kann man nur mit Vorbehalt von den Ionenströmen auf die Ionisierungsquerschnitte schließen, auch dann, wenn es sich nur um Relativwerte handelt. Das gilt insbesondere, wenn Ionen sehr verschiedener Massenzahl oder Anfangsenergie (kinetische Energie des Ions unmittelbar nach der Entstehung) miteinander verglichen werden sollen. Eine quantitative Aussage über den Ionisierungsquerschnitt werden wir also nur den Änderungen der auf ein Edelgas bezogenen Ionenströme mit der Temperatur zuschreiben können. Dementsprechend ist in den Abb. 3, 4, 5 und 6 die Größe $I_{\mathrm{M}}(T) / I_{\mathrm{E}}(T): I_{\mathrm{M}}\left(T_{0}\right) / I_{\mathrm{E}}\left(T_{0}\right)$ als Funktion der Temperatur aufgetragen. $I_{\mathrm{M}}(T)$ ist der Ionenstrom auf der Masse $M$ bei der Temperatur $T, I_{\mathrm{E}}(T)$ der Ionenstrom des gleichzeitig zugeführten Edelgases $\left({ }^{20} \mathrm{Ne}^{+}\right)$und $T_{0}$ die Bezugstemperatur. Ein gewisser apparativer Einfluß, dessen Größe sich schwer abschätzen läßt, bleibt trotzdem bestehen bei Ionen mit hoher Anfangsenergie, wenn sich diese Anfangs- energie stark mit der Temperatur ändert; und zwar bewirkt eine Zunahme der Anfangsenergie bei gleichbleibendem Ionisierungsquerschnitt eine Abnahme des Ionenstroms. Große Anfangsenergien ( $1 \mathrm{eV}$ ) sind vor allem bei kleinen Molekülbruchstücken zu erwarten, zum Beispiel bei $\mathrm{CH}_{3}{ }^{+}$-Ionen aus schweren Kohlenwasserstoffen. Beim Vergleich von Kohlenwasserstoffionen gleicher Kohlenstoffzahl ist die Anfangsenergie am größten bei den wasserstoffärmsten Ionen, wie Messungen von Taubert $^{9}$ gezeigt haben. Mit dieser Einschränkung bezüglich der Ionen großer und stark temperaturabhängiger Anfangsenergie bezeichnen wir die Meßgröße

$$
I_{\mathrm{M}}(T) / I_{\mathrm{E}}(T): I_{\mathrm{M}}\left(T_{0}\right) / I_{\mathrm{E}}\left(T_{0}\right)
$$

in den Abb. 3, 4, 5 und 6 als den auf die Temperatur $T_{0}$ bezogenen Ionisierungsquerschnitt für die Bildung des Ions $\mathrm{M}^{+}$.

\section{Meßergebnisse}

Es wurden die Massenspektren von neun gesättigten Kohlenwasserstoffen gemessen, und zwar die drei Pentane, die fünf Hexane und das $n$-Heptan. Zusammen mit früheren Messungen ${ }^{3}$ von $\mathrm{CH}_{4}$ bis $\mathrm{C}_{4} \mathrm{H}_{10}$ ergibt sich so ein guter Überblick über das Verhalten der gesättigten Kohlenwasserstoffe. Das Bild wird bei anderen Molekülen mit einer größeren Zahl von Atomen vermutlich ganz ähnlich sein. Wir zeigen nur eins der Spektren im Detail (Abb. 2) und beschränken uns im übrigen auf die Wiedergabe einiger charakteristischer Eigenschaften in den Abb. 3 bis 7 .

Die Änderung der Massenspektren mit der Temperatur läßt sich bei einer - wie wir glauben berechtigten - Extrapolation über den uns zugänglichen Temperaturbereich hinaus vereinfacht wie folgt beschreiben:

1. Der Ionisierungsquerschnitt aller Ionen eines Kohlenwasserstoffs $\mathrm{C}_{n} \mathrm{H}_{2 n+2}$ durchläuft mit zunehmender Temperatur ein Maximum. Das Maximum liegt um so tiefer, je schwerer das Ion ist. Einen Grenzfall bilden die Ionen der schwersten Kohlenstoffgruppe $C_{n}$, bei denen das Maximum des Ionisierungsquerschnitts erst am absoluten Nullpunkt erreicht wird, und deren Intensität um so schneller abfällt, je größer die Zahl der Kohlenstoffatome im

9 R. TAubert, Vortrag, gehalten auf der Tagung der Deutschen Physikalischen Gesellschaft 1957 in Heidelberg. 

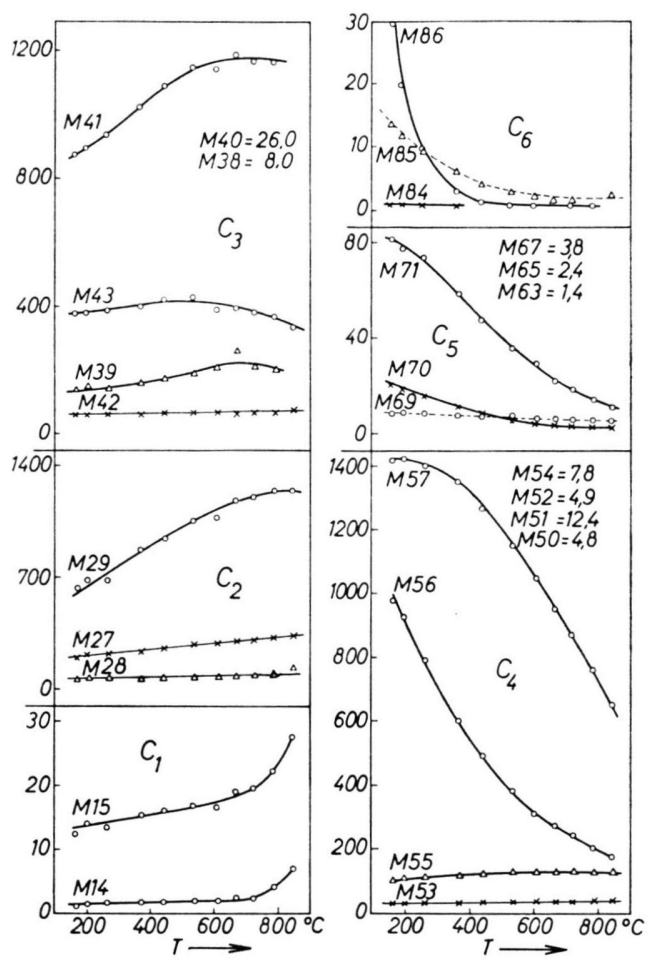

Abb. 2. Massenspektrum des 3-Methylpentans als Funktion der Temperatur, getrennt nach C-Gruppen. Als Ordinate ist aufgetragen der Ionenstrom auf den einzelnen Massen dividiert durch den Ionenstrom von ${ }^{20} \mathrm{Ne}^{+}$bei gleichem Partialdruck von Meßgas und Neon. Bei den über den ganzen Temperaturbereich konstanten Massenzahlen ist der Zahlenwert angegeben.

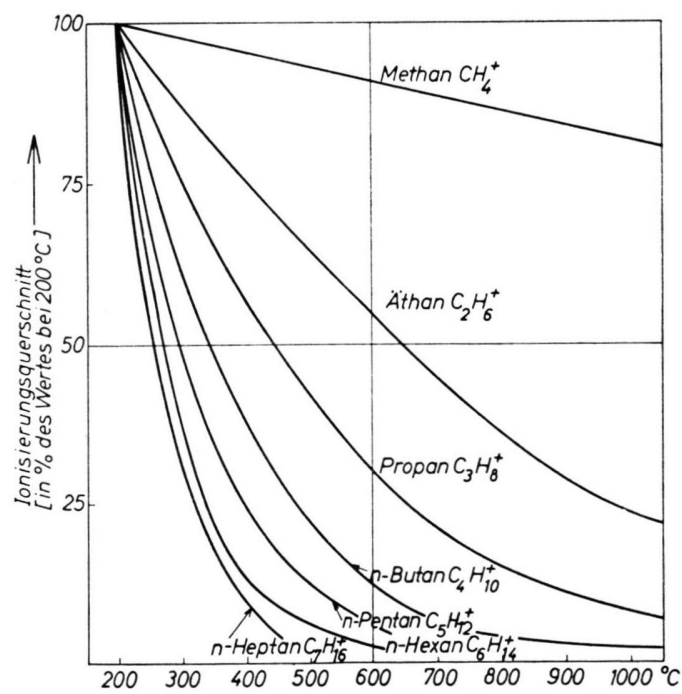

Abb. 3. Ionisierungsquerschnitte der „Mutterionen“ der geraden Kohlenwasserstoffe von $\mathrm{C}_{1}$ bis $\mathrm{C}_{7}$. Bezugstemperatur ist $200{ }^{\circ} \mathrm{C}$. Die Kurven für $\mathrm{C}_{1}$ bis $\mathrm{C}_{4}$ sind der Arbeit von $\mathrm{T}_{\mathrm{AUdBERT}}$ und Osberghaus ${ }^{3}$ entnommen.
Molekül ist (Abb. 3). Umgekehrt liegt bei den Ionen der Gruppe $\mathrm{C}_{1}$ das Maximum oberhalb von $1000{ }^{\circ} \mathrm{C}$.

2. Beim Vergleich von schweren Ionen gleicher Kohlenstoffzahl aber verschiedener Wasserstoffzahl ist die Temperaturabhängigkeit zwischen 100 und $1100{ }^{\circ} \mathrm{C}$ bei den wasserstoffreichsten Ionen im allgemeinen am größten. Ionen, die einen großen Teil ihrer Wasserstoffatome verloren haben, sind häufig nahezu konstant.

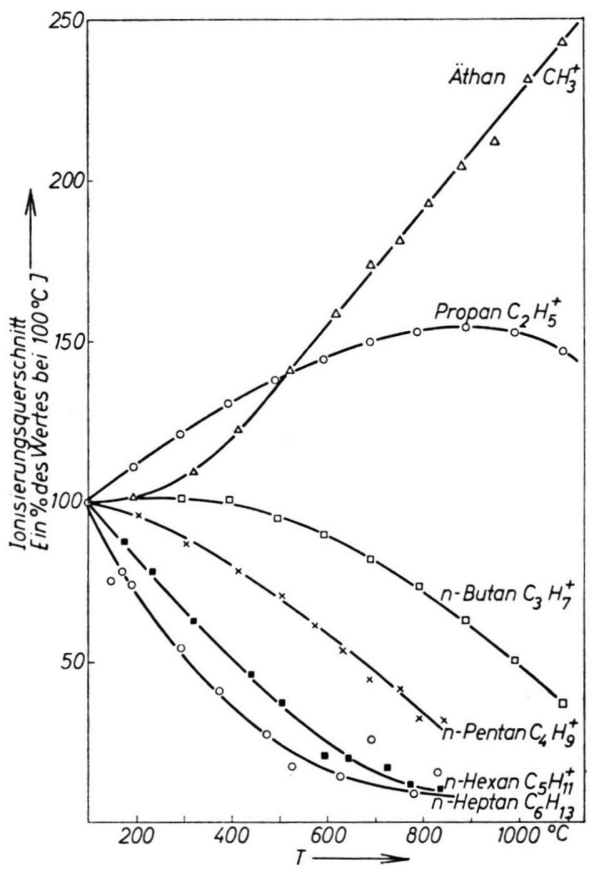

Abb. 4. Ionisierungsquerschnitte der aus den geraden Kohlenwasserstoffen $\mathrm{C}_{2}$ bis $\mathrm{C}_{7}$ durch Abspaltung einer $\mathrm{CH}_{3}$-Gruppe entstehenden Ionen. Bezugstemperatur ist $100{ }^{\circ} \mathrm{C}$. Die Kurven für Äthan, Propan und n-Butan sind der Arbeit von T $\mathrm{AU}$ bert und Osberghaus ${ }^{3}$ entnommen. Bei der starken Streuung der Meßwerte für n-Heptan oberhalb von $500{ }^{\circ} \mathrm{C}$ ist zu berücksichtigen, daß auf das Ion $\mathrm{C}_{6} \mathrm{H}_{13}{ }^{+}$weniger als $0,1 \%$ der Gesamtintensität entfällt. Ähnlich liegen die Verhältnisse beim n-Hexan.

3. Die Totalionisation, das heißt, die Summe aller Ionenströme, ist innerhalb der Meßgenauigkeit unabhängig von der Temperatur. Der in Abb. 7 bei einigen der Kohlenwasserstoffe zum Ausdruck kommende schwache Abfall mit zunehmender Temperatur kann durch Fehler in der Aufsummierung der Ionenströme entstanden sein. Diese Aufsummierung macht bei Temperaturen oberhalb von $600^{\circ} \mathrm{C}$ Schwierigkeiten, weil die beiden Massenzahlen 39 und 41 sehr stark mit Kalium-Ionen belegt sind, die aus dem Wandmaterial des Ionenkästchens stammen. 


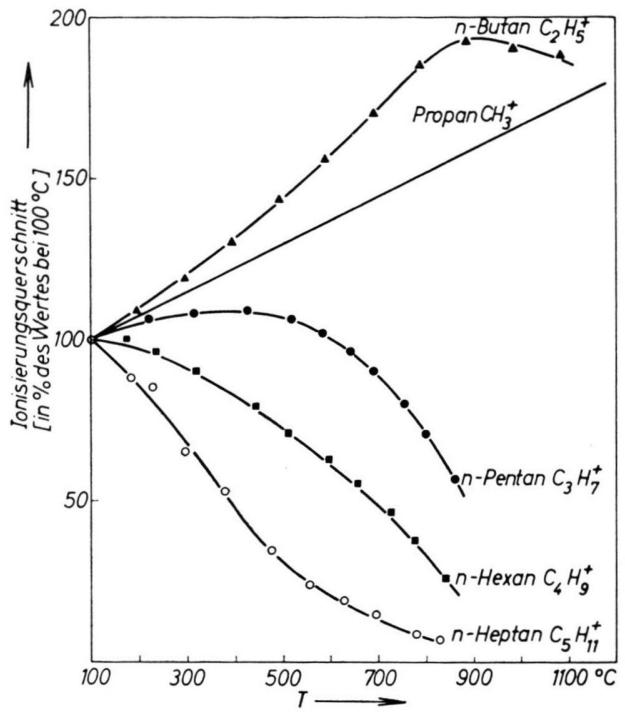

Abb. 5. Ionisierungsquerschnitte der aus den geraden Kohlenwasserstoffen $\mathrm{C}_{3}$ bis $\mathrm{C}_{7}$ durch Abspaltung einer $\mathrm{C}_{2} \mathrm{H}_{5}$-Gruppe entstehenden Ionen. Bezugstemperatur ist $100^{\circ} \mathrm{C}$. Die Kurven für Propan und n-Butan sind der Arbeit von TaUbert und Osberghaus ${ }^{3}$ entnommen.

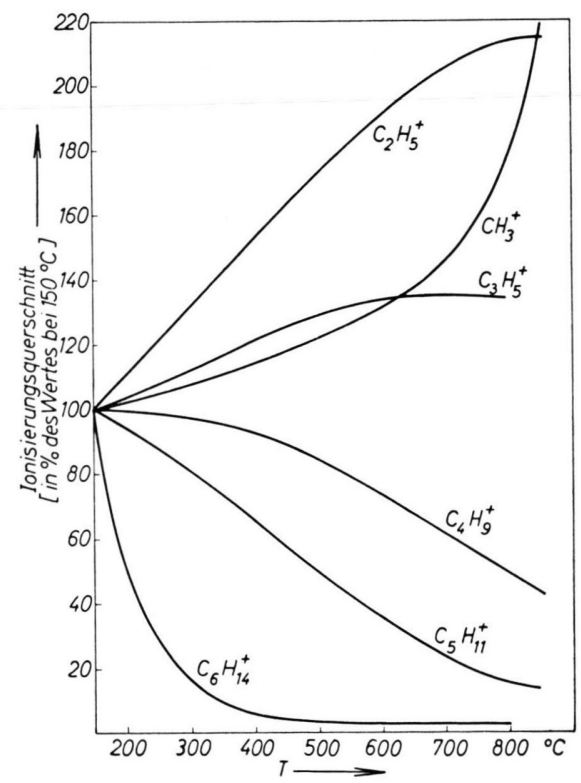

Abb. 6. Ionisierungsquerschnitte für die Ionen $\mathrm{CH}_{3}{ }^{+}, \mathrm{C}_{2} \mathrm{H}_{5}^{+}$, $\mathrm{C}_{3} \mathrm{H}_{5}{ }^{+}, \mathrm{C}_{4} \mathrm{H}_{9}^{+}, \mathrm{C}_{5} \mathrm{H}_{11}{ }^{+}$und $\mathrm{C}_{6} \mathrm{H}_{14}{ }^{+}$aus 3-Methylpentan; das sind jeweils die häufigsten Ionen in den einzelnen C-Gruppen. Bezugstemperatur ist $150^{\circ}$.

Die Totalionisation der fünf Hexane (möglicherweise mit Ausnahme des etwas abweichenden 2-Methylpentans) stimmt innerhalb der Meßfehler überein. Der Vergleich des Isopentans mit den Hexanen und dem $n$-Heptan zeigt, daß die Totalionisation etwa proportional zur Zahl der Kohlenstoffatome im Mole. kül ansteigt. Der Unterschied zwischen den beiden Pentanen Tetramethylmethan und Isopentan liegt außerhalb der Meßgenauigkeit. Es erscheint plausibel, daß der Ionisierungsquerschnitt des Tetramethylmethans wegen des gedrängten und nahezu kugelsymmetrischen Aufbaus des Moleküls kleiner ist als der der übrigen Pentane.

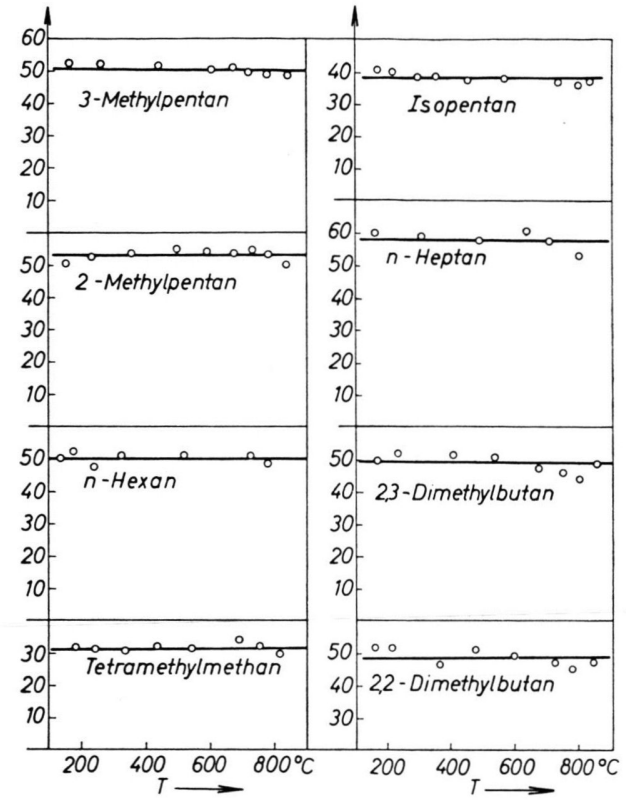

Abb. 7. Totalionisation in Abhängigkeit von der Temperatur. Ordinate ist der auf ${ }^{20} \mathrm{Ne}^{+}$bezogene Gesamtionenstrom.

\section{Messungen mit Katalysator-Einsatz}

Bei einem der Kohlenwasserstoffe (2,2-Dimethylbutan) wurden die Messungen mit einem Ionisierungskästchen wiederholt, dessen Wände mit einer $1 \mathrm{~mm}$ starken Schicht eines Perlkatalysators ausgelegt waren. Der Si-Al-Perlkatalysator W 700 wurde uns freundlicherweise von der Kalichemie AG., Hannover, zur Verfügung gestellt. Kontakte dieser Art werden in der Erdölindustrie als Crack-Katalysatoren verwendet. Das Spektrum wurde aufgenommen bis zur maximalen Arbeitstemperatur des Katalysators $\left(600{ }^{\circ} \mathrm{C}\right)$. Im Unterschied zu dem Ionisierungsraum mit Metallwänden zeigte sich ab $400{ }^{\circ} \mathrm{C}$ eine merkliche Methanbildung. Bei $560{ }^{\circ} \mathrm{C}$ wurde 1 Methanmolekül auf 50 Moleküle 2,2-Dimethylbutan gebildet (Abb. 8). Zwischenstufen der $\mathrm{CH}_{4}$-Bildung, vermutlich $\mathrm{CH}_{3}$-Radikale, konnten nicht gefunden wer- 
den. Aus der Temperaturabhängigkeit der Methankonzentration folgt für die Aktivierungsenergie der für die Methanbildung verantwortlichen Reaktion der Betrag von $25 \mathrm{kcal}$.

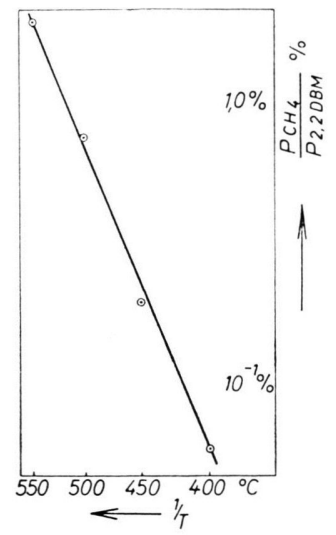

Abb. 8. Verhältnis der Partialdrucke von Methan und 2,2 Dimethylbutan in einem mit Perlkatalysator ausgekleidetem Ionisierungskästchen als Funktion der reziproken Temperatur (Druck im Ionisierungsraum $\approx 10^{-5}$ Torr).

\section{Deutung der Temperaturabhängigkeit der Spektren}

Die Bildung von Ionen durch Elektronenbeschuß eines Moleküls denkt man sich üblicherweise in zwei Schritte zerlegt. Zunächst wird das Molekül ohne Änderung der Kernabstände ionisiert: Übergang von der unteren Potentialkurve des Moleküls zur oberen Potentialkurve des Molekülions in Abb. 9. Je nach der Anfangslage der Atome und der Form der oberen Potentialkurve ist entweder das so entstandene Molekülion beständig, oder aber es erfolgt als zweiter Schritt ein Zerfall in kleinere Bruchstücke, von denen mindestens eins ionisiert ist. Im allgemeinen Fall eines $n$-atomigen Moleküls hat die Beschreibung in einem $3 n$-6-dimensionalen Potentialbild zu erfolgen, entsprechend den $3 n-6$ möglichen Normalschwingungen. Ein solcher Potentialtopf hat eine Reihe von "Ausflußrinnen“, die den verschiedenen Zerfallsmöglichkeiten des Moleküls bzw. Molekülions entsprechen. Diese Ausflußrinnen werden im allgemeinen um so höher liegen, je gröBer die Zahl der Spaltprodukte ist, zu denen sie führen. Die verschiedenen Zerfallsmöglichkeiten sind in Abb. 9, die einen Schnitt durch einen solchen Potentialkopf darstellen soll, schematisch angedeutet. Den Einfluß der Temperatur auf das Massenspektrum kann man ganz analog wie bei einem zweiatomigen Molekül verstehen ${ }^{3}$ : Abgesehen von den $\mathrm{C}-\mathrm{H}$ -
Schwingungen sind bei $1000{ }^{\circ} \mathrm{C}$ sämtliche Normalschwingungen der Kohlenwasserstoffe, auch die C - CStreckenschwingungen, schon merklich angeregt; das heißt, die von den $3 n-6$ Normalkoordinaten abhängige Schwingungseigenfunktion $\psi$ des Elektronengrundzustandes nimmt merkliche Werte außerhalb des Potentialminimums an. Mit zunehmender Temperatur wird also nach dem FRANCK-Condon-Prinzip ein immer größerer Prozentsatz der Übergänge bei der Inonisation zu Schwingungstermen des Molekülions führen, die höher liegen als eine oder mehrere der Ausflußrinnen: Das Molekülion zerfällt, und zwar werden zunächst größere Bruchstücke bevorzugt, die dann aber bei weiterer Temperatursteigerung gegenüber kleineren Molekülfragmenten zurücktreten. Das ist eine mögliche Erklärung für das Auftreten von Maxima in den Ionisierungsquerschnitten als Funktion der Temperatur. Vielleicht kann die relativ schwache Temperaturabhängigkeit von vielen der wasserstoffarmen Ionen dadurch erklärt werden, daß das Abspalten einer größeren $\mathrm{Zahl}$ von $\mathrm{H}$-Atomen die Anregung von $\mathrm{C}-\mathrm{H}$-Schwingungen voraussetzt, also erst bei Temperaturen oberhalb von $1000{ }^{\circ} \mathrm{C}$ eintritt.

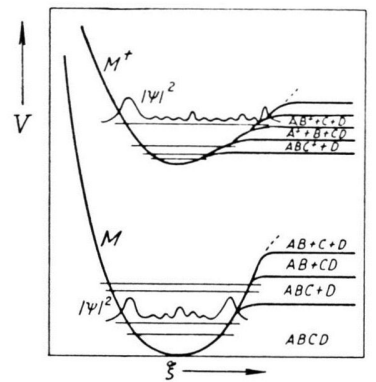

Abb. 9. Schematische Darstellung eines Schnittes durch den Potentialtopf eines mehratomigen Moleküls und Molekülions. Die verschiedenen von den Potentialkurven abbiegenden horizontalen Geraden sollen eine größere Zahl von Zerfallsmöglichkeiten und die dazu jeweils erforderliche Mindestenergie andeuten. In beide Potentialschnitte ist eine mehr oder weniger willkürliche Kurve für den Verlauf der Eigenfunktion $\left(|\psi|^{2}\right)$ in einem bestimmten Schwingungszustand eingezeichnet.

Die Konstanz der Totalionisation wird man bei dieser Zerlegung des Ionisierungsvorganges in zwei Schritte erwarten; man kann sie umgekehrt auch als einen indirekten Beweis für die Richtigkeit des Bildes ansehen, das man sich vom Ionisierungsprozeß macht. Die Temperaturunabhängigkeit des totalen Ionisierungsquerschnittes zeigt weiter, daß die Verfälschung der Messungen durch apparative Einflüsse nur geringfügig sein kann.

Wir danken Herrn Prof. Dr. W. Paul für die Unterstützung und Förderung dieser Arbeit. 